\title{
Validity, the Squeezing Argument and Alternative Semantic Systems: the Case of Aristotelian Syllogistic
}

\author{
Edgar Andrade-Lotero • Catarina Dutilh Novaes
}

Received: 12 January 2010 / Accepted: 19 November 2010 / Published online: 16 December 2010

(C) The Author(s) 2010. This article is published with open access at Springerlink.com

\begin{abstract}
We investigate the philosophical significance of the existence of different semantic systems with respect to which a given deductive system is sound and complete. Our case study will be Corcoran's deductive system $\mathrm{D}$ for Aristotelian syllogistic and some of the different semantic systems for syllogistic that have been proposed in the literature. We shall prove that they are not equivalent, in spite of $\mathrm{D}$ being sound and complete with respect to each of them. Beyond the specific case of syllogistic, the goal is to offer a general discussion of the relations between informal notions-in this case, an informal notion of deductive validity-and logical apparatuses such as deductive systems and (model-theoretic or other) semantic systems that aim at offering technical, formal accounts of informal notions. Specifically, we will be interested in Kreisel's famous 'squeezing argument'; we shall ask ourselves what a plurality of semantic systems (understood as classes of mathematical structures) may entail for the cogency of specific applications of the squeezing argument. More generally, the analysis brings to the fore the need for criteria of adequacy for semantic systems based on mathematical structures. Without such criteria, the idea that the gap between informal and technical accounts of validity can be bridged is put under pressure.
\end{abstract}

Keywords Syllogistic • Semantic systems for syllogistic • Proofs of completeness $\cdot$ Validity $\cdot$ Squeezing argument

\footnotetext{
E. Andrade-Lotero

Faculty of Natural Sciences and Mathematics, Universidad del Rosario,

Bogotá, D.C., Colombia

C. Dutilh Novaes $(\varangle)$

ILLC/Department of Philosphy, Universiteit van Amsterdam, Oude Turfmarkt 141-147, Amsterdam 1012 GC, Netherlands

e-mail: cdutilhnovaes@gmail.com
} 


\section{Introduction}

Let us start with a fairly uncontroversial observation. Generally speaking, a logical system can be viewed from (at least) two equally important but fundamentally different angles: i) it can be viewed as a pair formed by a syntax, i.e. a deductive system, and a semantics, i.e. a class of mathematical structures onto which the underlying language is interpreted; or ii) it can be viewed as a triad consisting of a syntax, a semantics and the target phenomenon that the logic is intended to capture. ${ }^{1}$ In the first case, both syntax and semantics are viewed as autonomous mathematical structures, not owing anything to external elements. In the second case, both syntax and semantics are accountable towards the primitive target phenomenon, which may be an informally formulated concept, or even phenomena in the 'real world' (e.g. logics of action, logics of social interaction, quantum logic etc.). Indeed, in the second case, both syntax and semantics seek to be a 'model' in some sense or another of the target phenomenon. In this paper, we will focus on logical systems viewed as triads syntax-semantics-target phenomenon. ${ }^{2}$

Against this background, we investigate the philosophical significance of the existence of different semantic systems that are 'matching' (in the sense of there being a proof of soundness and completeness) for a given deductive system. Our case study will be Corcoran's deductive system D for Aristotelian syllogistic and some of the different semantic systems for syllogistic that have been proposed in the literature. But the repercussions of the analysis go well beyond the case of syllogistic specifically. Rather, they concern the general topic of the relations between informal notions-in this case, an informal notion of deductive validity - and logical apparatuses such as deductive systems and (model-theoretic or other) semantic systems that aim at offering technical, formal accounts of informal notions.

More specifically, we will be interested in Kreisel's famous 'squeezing argument', which concerns precisely the relations between the three elements constituting a logic on the present approach (syntax, semantics and target phenomenon). We shall ask ourselves what a plurality of semantic systems

\footnotetext{
${ }^{1}$ In fact, in both cases one must also take into account the specific language being used, but for the sake of brevity we shall not consider the role played by the choice of a specific language for now (we will talk about it later though). Broadly speaking, the language may be considered as pertaining to the syntax.

${ }^{2}$ We here adopt a point of view similar to that of S. Shapiro: “... logic is, at root, a philosophical enterprise. Since at least the beginning of the twentieth century, however, logic has become a branch of mathematics as well as a branch of philosophy. [...] Our main question here concerns how that wonderful mathematics relates to the philosophical targets..."[21, 651] Let us also remind the reader that, in his seminal paper on logical consequence [26], Tarski also takes an informal notion of consequence as his starting point (encapsulated in his material conditions of adequacy: substitutivity and truth-preservation), and then goes on to give it a mathematical formulation.
} 
(understood as classes of mathematical structures) may entail for the cogency of specific applications of the squeezing argument. We shall argue that whenever there are reasons to think that the choice of a given semantics for a target phenomenon has not been adequately justified, the squeezing argument cannot straightforwardly go through. Indeed, the main thesis of the paper is that when there are alternative semantic systems available but with very different characteristics (as is the case of the systems analyzed here), they cannot all be equally adequate models of the target phenomenon. Relatedly, showing in what sense the four semantic systems for syllogistic analyzed here are not equivalent (in spite of system $\mathrm{D}$ being sound and complete with respect to each of them) is the main technical contribution of the paper.

In first instance, it might be thought that the discussion presented here does not generalize to logical systems other than syllogistic, given that the latter is an exceedingly simple and expressively weak deductive system. Indeed, it might be thought that it 'underdetermines' its own semantics precisely in virtue of its weak expressive power. But from the point of view of the present discussion, the expressive weakness of the deductive system itself is in fact irrelevant, as the goal is precisely to formulate a semantics that is an adequate model of the target phenomenon, and thus not a semantics to match the deductive system. More generally, the analysis brings to the fore the need for criteria of adequacy for semantic systems based on mathematical structures that aim at capturing a given target phenomenon-e.g. an informal concept of validity. Without such criteria, the idea that the gap between conceptual and technical accounts of validity can be bridged is put under pressure. ${ }^{3}$

The paper proceeds as follows. We start in Section 2 with a discussion of Aristotle's goals and results in the Prior Analytics and of Corcoran's interpretation of Aristotle's enterprise. In Section 3 we discuss Kreisel's squeezing argument and possible reasons why it may not go through in specific cases. In Section 4 we present: (i) Corcoran's deductive system D; (ii) a general framework that allows us to compare the different semantic systems for syllogistic; and (iii) a discussion of inclusion relations between these different systems; in particular, we show that they are not extensionally equivalent. Finally, in Section 5 we argue that this extensional disagreement suggests that they cannot all be equally adequate semantic systems for syllogistic; we also comment on Shapiro's and Etchemendy's respective views on the philosophy of modeltheory. In conclusion, we argue that these issues jeopardize the cogency of any instantiation of the squeezing argument whenever the legitimacy of a given semantics for a logic has not been sufficiently established.

\footnotetext{
${ }^{3}$ We do not mean to suggest that there should be a principled set of necessary and sufficient conditions of adequacy for semantic systems, to be applied 'across the board'; to some extent, specific cases will require specific discussions. But we do think that general guidelines are required, which in turn should reflect our goals when designing a semantics for a logic.
} 


\section{Aristotle and Corcoran}

\subsection{Aristotle on Syllogistic}

At the very beginning of the Prior Analytics, that is before presenting the details of his deductive system, Aristotle presents a much-discussed general definition of deduction:

A deduction is a discourse in which, certain things having been supposed, something different from the things supposed results of necessity because these things are so. By 'because these things are so' I mean 'resulting through them', and by 'resulting through them' I mean 'needing no further term from outside in order for the necessity to come about' (Aristotle, Prior Analytics, 24b19-23, quoted from [25]).

An important feature of this general definition is that, although it makes reference to a discourse, and thus a fortiori to a language, it in fact suggests that whether a thing stated in a language 'results of necessity' from other things being stated in the same language is independent of the peculiarities of the language in question. ${ }^{4}$ In particular, as has often been noticed in the literature, ${ }^{5}$ this definition is not restricted to the particular class of arguments which Aristotle is effectively dealing with throughout the Prior Analytics, namely arguments composed of two premises and one conclusion, all of which are sentences of the $a, i, e$ or $o$ forms (also known as categorical sentences).

Immediately after presenting the general definition of a deduction, Aristotle introduces the distinction between perfect and imperfect arguments/deductions.

I call a deduction [perfect] ${ }^{6}$ if it stands in need of nothing else besides the things taken in order for the necessity to be evident [emphasis added]; I call it [imperfect] if it still needs either one or several additional things which are necessary because of the terms assumed, but yet were not taken by means of premises (Aristotle, Prior Analytics, 24b24-27, quoted from [25]).

Roughly put, a deduction is perfect if the validity of its inferential steps is immediately evident (think of how one decomposes a proof in all its immediate steps), and imperfect otherwise. Notice that, according to the general definition of deduction, imperfect deductions are just as valid as perfect deductions: the conclusion 'results of necessity' from the premises in just the

\footnotetext{
${ }^{4}$ It is also independent of the contingency of an agent actually performing a deduction. It is in this sense that it can be said (as Corcoran and others emphasize) that for Aristotle the validity of an argument is a matter of fact, not an epistemic matter.

${ }^{5}$ For example, [25, pp. 109-110].

${ }^{6}$ The Smith translation, which we follow here, has 'complete' and 'incomplete', but we prefer to stick to the more traditional 'perfect' vs. 'imperfect' terminology precisely because of its familiarity.
} 
same way (although of course there are different possible interpretations to the 'necessity' in question). The difference between perfect and imperfect deductions is an epistemic one, related to whether their validity is made evident to us.

Therefore, in order to account for a limited class of valid arguments, namely the valid categorical arguments (which are traditionally referred to as 'syllogisms'), in the Prior Analytics Aristotle proves their validity on the basis of deductive steps whose validity is (or so he claims) immediately apparent; this procedure is referred to as 'perfecting' or 'completing' imperfect syllogisms. These evidently valid deductive steps are: the four 'perfect syllogisms' in the first figure (Barbara, Celarent, Darii and Ferio, according to the medieval terminology); conversion (from 'Some $x$ is $y$ ' infer 'Some $y$ is $x$ ', and from 'No $x$ is $y$ ' infer 'No $y$ is $x$ '); and subalternation (from 'All $x$ is $y$ ' infer 'Some $x$ is $\left.y^{\prime}\right) .{ }^{8}$ The basic procedure is to assume the premises of an imperfect syllogism, and by means of successive applications of the deductive steps listed above, to reach the desired conclusion, i.e. the conclusion of the syllogism being perfected. ${ }^{9}$ The underlying assumption is clearly that a chain of valid deductive steps constitutes a valid deduction. Importantly, though, the deduction of an imperfect syllogism is not intended to validate the imperfect syllogism in question; rather, it is intended to make its validity (which is a factual property of an argument) evident to us.

\subsection{A Problem of Completeness}

Hence, Aristotle's general project in the Prior Analytics is essentially that of 'perfecting' valid syllogisms. However, a problem that presents itself immediately, but which is not explicitly addressed by Aristotle, is whether these deductive rules are sufficient to deduce, and thus to show as valid, all categorical arguments which are valid according to the definition of a valid argument at the beginning of the text. Can every imperfect but nevertheless valid syllogism be shown to be valid by means of a succession of such inferential steps? In other words, a problem of deductive completeness presents itself for the Aristotelian project of showing that all valid syllogisms can be perfected (and thereby their validity made evident to us).

\footnotetext{
${ }^{7}$ From now on, we will use the traditional terminology, employing the term 'syllogism' to refer to arguments of this specific class. But the reader must always bear in mind that the general definition of deduction is not meant to cover only valid arguments in this class.

${ }^{8}$ These deductive steps are formally presented in Section 4.1. Aristotle proves the validity of conversions (25a14-26), but as for the perfect syllogisms, he seems to suggest that their validity is evident on the basis of the principles 'predicated-of-all' and 'predicated-of-none' formulated in 24b27-31. Subalternation is related to existential import. Moreover, he sometimes (e.g. with Baroco and Bocardo) needs to use an indirect proof to perfect a syllogism.

${ }^{9}$ See [3, Section 4.1].
} 
The problem of the deductive completeness of Aristotle's syllogistic system thus formulated is addressed by J. Corcoran in a series of papers. ${ }^{10}$ Corcoran develops a fomalization of Aristotle's syllogistic based on a natural deduction system D and on a semantic system consisting of families of non-empty sets. He proves that system D is sound and complete with respect to this semantic system, ${ }^{11}$ and the completeness proof is the (purported) solution to the problem of deductive completeness. The assumption seems to be that the semantics of families of non-empty sets is able to accurately capture, at least extensionally, the underlying notion of validity for this restricted class of arguments. Whatever argument that is valid according to this semantic system is valid tout court, and moreover the semantics is (presumably) exhaustive in that it deems as valid all and only the arguments of the restricted language which are indeed valid according to the underlying notion. So a proof of completeness with respect to this semantic system would ensure that D is deductively complete, i.e. that it can show to be valid exactly those arguments in this fragment of the language that are indeed valid.

\subsection{Choosing a Semantics}

But why is it that this particular semantic system provides the right extension to the underlying notion of validity (for the categorical fragment of the language)? Of course, the former is a representation of the latter, with a fair amount of simplification-something that Corcoran himself is well aware of-and yet he seems to consider it to be an uncontroversial interpretation for syllogistic onto mathematical structures. ${ }^{12}$ But clearly, the adequacy of this representation (even if it is only meant to capture the extension of an underlying notion of validity) can be brought into question. The adequacy of the semantic system is as much in need of justification as is that of deductive system D. If we lack sufficient justification for its adequacy, then it is not entirely obvious what is accomplished from a philosophical point of view by means of a proof of completeness such as the one formulated by Corcoran.

In effect, the need to offer adequate justification for the choice of a given semantic system for syllogistic becomes more pressing in view of the alternatives available in the literature, in particular the three other semantic systems for syllogistic that we discuss in Section 4. Given that the semantic system is summoned to provide an accurate account (at least extensionally) of the underlying (and presumably unique) informal notion of validity for this fragment of the language, then the point is pressing when there are competing

\footnotetext{
${ }^{10}$ See $[6,7]$ and Section 4.1 below. Notice that Smiley [22] put forward a similar interpretation of Aristotelian syllogistic almost simultaneously, which was developed independently of Corcoran's investigations.

${ }^{11}$ See [5].

${ }^{12}$ We shall say much more on the issue of the status of semantic systems as 'models' in Section 5, in connection with Etchemendy's and Shapiro's respective views on the matter.
} 
semantic systems with remarkably different characteristics. System D is sound and complete with respect to each of them, and they are each conceptually motivated on independent grounds (see Section 4). So how do we ascertain that a given semantic system is indeed successful in capturing the extension of the underlying general notion of validity, so as to serve as a yardstick to evaluate the adequacy of the deductive system with respect to the target phenomenon ${ }^{13}$

It may seem that the existence of at least four different semantic systems for syllogistic is not in itself particularly troublesome, that is if these different semantic systems are all 'equivalent', in a relevant sense of 'equivalent'. But we will show that the apparent equivalence between these systems (given that $\mathrm{D}$ is sound and complete with respect to each of them) depends crucially on the limited expressive power of the language underlying D. ${ }^{14}$ As soon as one considers languages with more expressive power (with appropriate interpretations of the additional terminology in each of these classes of structures), one realizes that these semantic systems do not extensionally coincide-that is, they do not deem as valid the exact same class of arguments in the extended languages. And given that the underlying informal notion of validity is intended to be perfectly general, an extensional disagreement between the different semantic systems in extended languages would suggest that they could not all have been equally adequate semantic systems even for the original language.

In summary, Corcoran's completeness proof will only have the philosophical significance he attributes to it if there are compelling (independent) reasons to believe that the semantic system in question is an adequate representation of the underlying informal notion. But as we shall argue, the availability of alternative semantic systems forces us to evaluate the adequacy claim more carefully.

\section{The Squeezing Argument}

\subsection{A Schematic Version of the Squeezing Argument}

The issue of the philosophical significance of proofs of (soundness and) completeness is an important one, and illustrious philosophers/logicians such as Dummett [9] and Kreisel [13] have given positive answers to the question whether such technical results have philosophical significance at all. Kreisel, in particular, offered an argument intended to show that completeness proofs (at

\footnotetext{
${ }^{13}$ Syllogistic is just one specific case of this issue, and by discussing this relatively simple case we hope to bring the point home that the lack of adequacy criteria might be a problem for several other semantic analyses based on mathematical structures.

${ }^{14}$ In this paragraph we advance some of the claims we will be arguing for in more detail throughout the paper.
} 
least in some cases) could be seen as bridging the gap between informal and technical notions of validity: the much-discussed 'squeezing argument' ${ }^{15}$

Corcoran does not refer to Kreisel explicitly, but we think that his defense of the adequacy of deductive system $\mathrm{D}$ on the basis of a completeness proof can be viewed as a variation of Kreisel's squeezing argument. ${ }^{16}$ So here is an outline of the argument, following its presentation in [23]. ${ }^{17}$ Consider a theoretically robust but informally formulated concept I ('I' for 'informal', not for 'intuitive'), typically with somewhat vague borders. Assume that there is a technical, formal and precisely defined concept $\mathrm{D}$, which can be predicated of (roughly) the same category of entities $e$ as I, and which, as it turns out, seems to offer sufficient conditions for an entity to fall under I. Thus:

- If $e$ is $\mathrm{D}$, then $e$ is $\mathrm{I}$.

Assume moreover that there is another technical, formal and precisely defined concept $\mathrm{S}$ which uncontroversially provides necessary conditions for something to fall under I. Thus:

- If $e$ is I, then $e$ is $\mathrm{S}$.

Given these two implications, "the extension of I (vaguely gestured at and indeterminately bounded though that might be) is at least sandwiched between the determinately bounded extensions of [D] and [S]" [23]. Now, what makes squeezing arguments truly interesting is that, in some cases, there is a third implication whose truth can be established by means of a proof (given that D and $\mathrm{S}$ are technical, mathematically defined concepts):

\section{- If $e$ is $\mathrm{S}$, then $e$ is $\mathrm{D}$.}

Given this third implication, the informal concept I is 'squeezed' between the two technical concepts, i.e. its extension is shown to coincide with that of the other two concepts. We thereby obtain a precise, technical formulation of the boundaries of informal, vague but crucial concept I.

\footnotetext{
${ }^{15}$ Recent discussions of it can be found in [11, especially Section 2.3], [24, ch. 35] and [23]. Notice that Kreisel himself does not use the phrase 'squeezing argument'.

${ }^{16}$ To be sure, although we will now raise objections to Kreisel's argument, we find much to commend in his analysis. For starters, he recognizes that the most fundamental level when dealing with the matter of validity is the informal, non-technical level, and that technical accounts aim at capturing underlying non-technical notions, i.e. the target phenomena.

${ }^{17}$ Smith is here openly criticizing Field's rendition of the squeezing argument in [11, Section 2.3]. In particular, Smith criticizes Field's idea of an 'intuitive', non-theoretical notion of validity, a criticism we entirely endorse. The problem with the 'intuitive' terminology used in connection with the squeezing argument by Field, and in fact more generally in philosophy of logic (e.g. by Etchemendy), is that it overlooks the fact that the initial target phenomenon, as we call it, is in effect already couched on a significant amount of theorizing, albeit of the 'informal' kind.
} 


\subsection{The Squeezing Argument and Validity}

It should be clear by now that, when it comes to logical systems conceived as triads, the informal concept I is precisely the target phenomenon that we take both syntax and semantics to be (attempted) models of. Whether syntax or semantics can be viewed as necessary or sufficient conditions specifying the borders of the target phenomenon (in specific cases) is of course the crux of the matter; it is the issue of how good a model they are of their target phenomenon.

The squeezing argument is perfectly schematic (as clearly shown in Smith's presentation), and different concepts can be used to instantiate it. (For example, in [24, ch. 35], it is used in connection with the concept of computability.) It is in particular often presented in connection with informal conceptions of validity and their technical counterparts (deductive systems and semantic systems). For instance: assume an informal notion of validity (for short, Ivalidity); a notion of validity as derivability in a given deductive system (for short, D-validity); and a notion of validity defined by a given technical semantic apparatus, model-theoretic or otherwise (for short, S-validity). The appeal of the squeezing argument here lies on the purported intuitiveness of two premises (the second one is the contrapositive of the second implication above, which spells out the necessary conditions for informal concept I). Let $K$ be a set of sentences and $d$ be a sentence (in a given language):

(1) If an argument from $K$ to $d$ is D-valid, then the argument is I-valid.

(2) If an argument from $K$ to $d$ is not $\mathrm{S}$-valid, then the argument is not I-valid.

Moreover, it relies on a technical result (if available) for the particular deductive system and the particular semantic system in question, namely a proof of completeness:

(3) (Completeness) If the argument from $K$ to $d$ is S-valid, then the argument is D-valid.

From (1)-(3) it follows that an argument is S-valid iff it is I-valid iff it is D-valid.

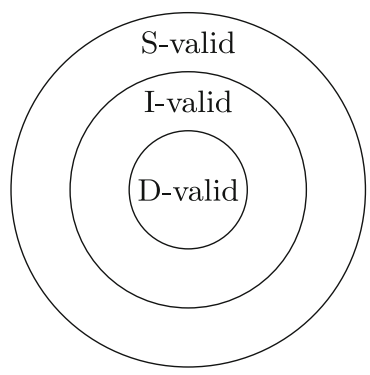

The idea underlying the two premises (1) and (2) seems to be that the syntactical approach (derivability) is particularly suitable for capturing (an informal notion of) validity, while the semantic approach is particularly suitable 
for capturing (an informal notion of) invalidity. ${ }^{18}$ This observation is supported by the quantificational asymmetry between the syntactical and the semantic notions. That is, from the syntactical point of view, validity corresponds to an existential claim (there is a derivation from $K$ to $d$ ), while invalidity corresponds to a (negative) universal claim (no derivation is a derivation from $K$ to $d$ ). In contrast, from a semantic point of view, validity corresponds to a universal claim (all interpretations satisfying $K$ also satisfy $d$ ) while invalidity corresponds to an existential claim (there is an interpretation that satisfies $K$ but does not satisfy $d$ ). Now, insofar as, given our limited cognitive capacities, dealing with existential claims is far more manageable than dealing with universal claims, it seems prima facie plausible that there is indeed a tight connection between the notions of validity and derivability, on the one hand, and invalidity and counterexamples, on the other hand. And if it is indeed so, then premises (1) and (2) may appear to be beyond reproach. Here, we shall focus on (2) and argue that, at least in some important cases, it is not nearly as uncontroversial as one might think. ${ }^{19}$

Kreisel [13, p. 154] himself does not offer abundant motivation for (his version of) (2). He merely says: "on the other hand one does accept $[(2)]^{20}$ the moment one takes for granted that logic applies to mathematical structures." But there are two subtle implications in this assertion: (i) Which mathematical structures should a given (informal notion of) logic apply to? Does any mathematical structure fit the bill? Obviously not, but what criteria can be used to determine the appropriate mathematical structure(s) in each case? Naturally, there are quite a few different classes of mathematical structures available: in this paper alone, we consider families of non-empty sets, lattices, pairs of numbers and first-order models, and it is not at all obvious that the choice among different alternatives will typically be a straightforward matter. We shall argue that it is by no means straightforward in the case of syllogistic. (ii) Even more fundamentally, why should we take for granted that 'logic' (whatever it is that Kreisel means by 'logic' here) applies to mathematical structures? This is a substantive claim that would require further support. It seems to us that the idea of developing logic in connection with mathematical structures is essentially related to the logicist project of providing logical foundations to mathematics, and it is not obvious that this assumption should hold irrestrictly also beyond the scope of the logicist program. After all, deductive validity is a notion that goes well beyond its possible interpretations onto mathematical structures.

Kreisel seems to have in mind cases where the 'obvious' mathematical structure presents itself (he focuses explicitly on mathematics and mathematical logic in his analysis). In the case of axiomatizations of arithmetic, for example,

\footnotetext{
${ }^{18}$ This observation is explicitly found in e.g. [9, p. 292], and [8, p. 262].

${ }^{19} \mathrm{We}$ do not mean to imply that (1) is itself uncontroversial, but our focus here is on the connection between target phenomena and semantic systems.

${ }^{20} \mathrm{Kreisel}$ 's version of (2) is a direct implication (as also in Section 3.1 above), not a contrapositive: in his notation, $\forall i \forall \alpha \mathrm{Val} \alpha^{i} \rightarrow V \alpha^{i}$. Nevertheless, his formulation and (2) are clearly equivalent.
} 
a mathematical structure (the structure of the natural numbers) is the very starting point for the analysis, i.e. its target-phenomenon, and the goal is to provide a syntactical (deductive) description of it by means of an axiomatization. In such cases, obviously there is an adequate interpretation onto mathematical structures for the axiomatic system, namely the very structure that was taken as a starting point. However, in cases where it is not obvious which mathematical structures (if any) should be used to provide a technical-semantic account of an informal notion (of validity or otherwise), the intuitive plausibility of (2) is considerably weakened. ${ }^{21}$

\subsection{Syllogistic and the Squeezing Argument}

Of course, the starting point for the syllogistic deductive system (both in Aristotle's own 'semi-formal' presentation and in Corcoran's formalization) is not a mathematical structure. But what is the target phenomenon in this case? In other words, what corresponds here to the informal notion of validity, i.e. I-validity? This is a crucial question to be asked. We follow P. Smith [23] on the idea that this informal notion will not be 'pre-theoretical' in any way; it will already be a theoretically robust (albeit informal) notion. As we see it, there are two main candidates: the first candidate is the notion of validity encapsulated in Aristotle's general definition of a deduction quoted above; the second candidate is the collection of principles and results formulated throughout the Prior Analytics. But we maintain (following the interpretation of scholars such as R. Smith) that the second candidate is itself already an attempt at a (semiformal) systematization of the first candidate for a restricted fragment of the language. Therefore, the actual target phenomenon is ultimately the general definition of a deduction formulated at the very beginning of the text, in terms of what follows 'of necessity'. The theory presented throughout the Prior Analytics corresponds already to a semi-technical rendition of this notion.

As we interpret it, Corcoran's argument to the effect that Aristotle's syllogistic is deductively complete has a slightly different structure from a classical 'squeezing argument'. It is meant to show (a):

(a) If an argument from $K$ to $d$ is I-valid, it is D-valid.

But his proof only delivers the following:

(b) If an argument from $K$ to $d$ is S-valid, it is D-valid.

To go from (b) to (a), premise (2) of the squeezing argument (or something equivalent to it) is necessary, that is: "If an argument from $K$ to $d$ is I-valid, then it is S-valid."

\footnotetext{
${ }^{21}$ Thus, we concede that, in cases where there are compelling arguments supporting the choice of a given class of mathematical structures as a semantics for an informal notion, the squeezing argument may well go through. The bone of contention then becomes when this is indeed the case.
} 
So in this case the job of the completeness proof is more specifically to prove the adequacy of the deductive system, i.e. D-validity. But as suggested, the adequacy of the semantics that Corcoran chooses for his formalization cannot be taken for granted, i.e. it cannot be taken for granted that it perfectly captures (even if only extensionally) the underlying notion of validity for the categorical fragment of the language. ${ }^{22}$ Now, if there aren't sufficient grounds to be confident that a given technical notion of validity (in terms of a semantics onto mathematical structures) successfully captures the necessary conditions for the informal target concept, the corresponding instantiation of premise (2) is not in any way self-evident. This holds in particular of syllogistic, which does not have an obvious candidate for S-validity. ${ }^{23}$

That syllogistic does not have an evident candidate for S-validity so as to allow for the squeezing argument to go through will become patent in the next section, when we discuss four different semantics for syllogistic that have been proposed in the literature. Each of them is substantially different from the others and yet independently motivated. How does one choose between alternative semantic systems for a given target concept? They cannot all be equally adequate.

\section{Semantic Systems}

\subsection{Corcoran's System}

Corcoran introduces Aristotelian syllogistic along the lines of familiar presentations of modern logical systems, i.e. in terms of a formal language, a deductive system and a semantic system. ${ }^{24}$ We will discuss them in turn, but notice that instead of one, we will present four different semantic systems.

The vocabulary of the formal language consists of a set $R:=\{a, e, i, o\}$, and a non-empty set $V$. The set of categorical sentences, $\mathcal{P}_{V}$, consists of those and

\footnotetext{
${ }^{22}$ Corcoran seems to go from 'valid' tout court to 'semantically valid'."[T] he Aristotelian system is seen to be complete in the sense that every valid argument expressible in his system admits of a deduction within his deductive system; i.e. every semantically valid argument is deducible" [7, p. 85, emphasis added].

${ }^{23} \mathrm{R}$. Williams discusses difficulties with (2) for some nonclassical logics in a blog post dated May 15th 2008, available at http://theoriesnthings.wordpress.com/2008/05/15/squeezing-arguments/. He says: "Kreisel gave a famous and elegant argument for why we should be interested in modeltheoretic validity. But I'm not sure who can use it." We argue here that the logician interested in syllogistic is not one of those who can use it. In a sense, the squeezing argument cannot help you precisely when you need it most, i.e. in cases where it is unclear what semantic system, if any, is adequate for the task at hand (e.g. some non-classical logics, as discussed by Williams).

${ }^{24}$ The present exposition of Corcoran's system is adapted from [5, 7].
} 
only those expressions of the form $x a y, x e y, x i y$, xoy where $x, y \in V$, and where $x \neq y .^{25}$

In the language of categorical sentences there is no propositional negation; the key notion is the semantic notion of contradiction. We assume a (meta-) function called the contradictory of function, denoted by $c: \mathcal{P}_{V} \rightarrow \mathcal{P}_{V}$, defined as follows:

$$
c(d):= \begin{cases}x o y & \text { if } d=x a y, \\ x i y & \text { if } d=x e y, \\ x e y & \text { if } d=x i y, \\ x a y & \text { if } d=x o y\end{cases}
$$

It is easy to see that $c(c(d))=d$ for every $d \in \mathcal{P}_{V}$.

The deductive system consists of the following natural deduction rules: ${ }^{26}$
(I) $\frac{x e y}{y e x}$
(II) $\frac{x a y}{x i y}$
(III) $\begin{aligned} & z a y \\ & \frac{x a z}{x a y}\end{aligned}$
(IV) $\begin{aligned} & z e y \\ & \frac{x a z}{x e y}\end{aligned}$

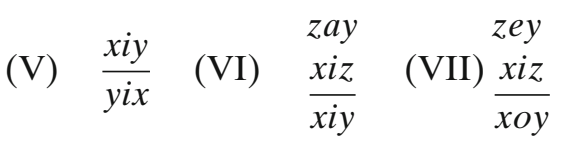

Definition 1 We say that a sequence $\left\langle p_{1}, \ldots, p_{n}\right\rangle$ of categorical sentences is a direct deduction of $d$ from $K$ if $d=p_{n}$ and for each $i \in\{1, \ldots, n\}$ one of the following holds:

1. $p_{i} \in K$, or

2. There exists $j<i$ such that $p_{i}$ is obtained from $p_{j}$ using rules (I), (II), or (V), or

3. There exist $j, k<i$ such that $p_{i}$ is obtained from $p_{j}$ and $p_{k}$ using (III), (IV), (VI), or (VII).

\footnotetext{
${ }^{25}$ This last requirement is actually important, both from a historical and from a mathematical perspective. First, it is a moot point between Corcoran and Łukasiewikz [15] whether Aristotle actually considered reflexive sentences, also known as self-predication, in his syllogistic systeme.g. xax. According to Corcoran, throughout Aristotle's works we can find only one use of negative reflexive sentences; Aristotle never discusses positive reflexive sentences [7, p. 99]. Moreover, if such reflexive sentences are allowed, the natural deduction system will not be complete with respect to the interpretation of the categorical sentences onto families of non-empty sets (see footnote 27).

${ }^{26}$ Rules (I) and (V) are known in the tradition as the conversion rules; Rule (II) is known as subalternation; Rules (III), (IV), (VI), and (VII) are known as the perfect syllogisms-in the medieval terminology, Barbara, Celarent, Darii and Ferio respectively.
} 
Definition 2 We say that a sequence $\left\langle p_{1}, \ldots, p_{n}\right\rangle$ of categorical sentences is an indirect deduction of $d$ from $K$ if there exists a $j<n$ such that $p_{n}=c\left(p_{j}\right)$ and for each $i \in\{1, \ldots, n\}$ one of the following holds:

1. $p_{i} \in K+c(d)$, or

2. There exists $j<i$ such that $p_{i}$ is obtained from $p_{j}$ using rules (I), (II), or (V), or

3. There exist $j, k<i$ such that $p_{i}$ is obtained from $p_{j}$ and $p_{k}$ using (III), (IV), (VI), or (VII).

We say that $K \vdash_{D} d$ if and only if there exists either a direct or an indirect deduction of $d$ from $K$. The logical system so defined is called D. It is a well-known fact (Aristotle himself seemed to be aware of it) that system D is redundant; for example, it can be proved that $\mathrm{D}$ is indeed equivalent to a much simpler system which consists only of rules (I)-(IV), which Corcoran named system RD.

On the present account, semantic systems for syllogistic are based on the notion of interpretation functions. In turn, an interpretation function is based on two elements: (i) a set $M$, and (ii) a function $g: V \rightarrow M$. An interpretation is defined as a function $\llbracket \cdot \rrbracket_{M, g}: \mathcal{P}_{V} \rightarrow\{0,1\}$ that assigns a truth-value to every categorical sentence based on the terms in $V$, according to their interpretations under $g$. It does so by defining when the value of a sentence under it is 1 :

1. $\llbracket x a y \rrbracket_{M, g}=1$ if and only if $g(x) \mathbf{A} g(y)$ holds,

2. $\llbracket x e y \rrbracket_{M, g}=1$ if and only if $g(x) \mathbf{E} g(y)$ holds,

3. $\llbracket x i y \rrbracket_{M, g}=1$ if and only if $g(x) \mathbf{E} g(y)$ does not hold,

4. $\llbracket x o y \rrbracket_{M, g}=1$ if and only if $g(x) \mathbf{A} g(y)$ does not hold.

In other words, $\llbracket \cdot \rrbracket_{M, g}$ says that xay is true if and only if some relation $\mathbf{A}$ between $g(x)$ and $g(y)$ holds. It also says that xey is true if and only if some other relation $\mathbf{E}$ between $g(x)$ and $g(y)$ holds. The rest of the definition follows from the requirement that $a$ and $o$, and $e$ and $i$ be contradictory sentences, respectively. Given that the interpretation is based on these relations, we will sometimes write $\llbracket \cdot \rrbracket_{M, g}^{\mathbf{A}, \mathbf{E}}$ to emphasize this dependency. But before we formulate the general definition of a semantic system for $\mathrm{D}$, a few more steps are required.

Let $d \in \mathcal{P}_{V}, K \subseteq \mathcal{P}_{V}$, and let $\llbracket \llbracket \rrbracket_{M, g}$ be an interpretation function:

- $\quad$ If $\llbracket d \rrbracket_{M, g}=1$, we say that $\llbracket \cdot \rrbracket_{M, g}$ is a true interpretation of $d$.

- We say that $\llbracket \cdot \rrbracket_{M, g}$ is a true interpretation of $K$ if $\llbracket \cdot \rrbracket_{M, g}$ is a true interpretation of $d$, for each $d \in K$.

- With respect to a class $\mathcal{C}$ of interpretation functions, $K \models_{\mathcal{C}} d$ if and only if for every interpretation function $\llbracket \cdot \rrbracket_{M, g}$ in $\mathcal{C}$, if $\llbracket \cdot \rrbracket_{M, g}$ is a true interpretation of $K$, it is also a true interpretation of $d$.

A semantic system for $\mathrm{D}$ is a class of interpretation functions. Here, we are only interested in specific classes, namely, the matching classes of interpretation 
functions for D. A matching class $\mathcal{C}$ of interpretation functions for $\mathrm{D}$ is a class of interpretation functions such that $\mathrm{D}$ is (strongly) sound and complete with respect to it, that is, such that for all $K$ and $d, K \vdash_{D} d$ iff $K \models_{\mathcal{C}} d$.

In the next sections we discuss a number of examples of classes of interpretation functions with these characteristics, and we shall also look into the structure among these classes.

\subsection{Different Semantics for D}

\subsubsection{Corcoran's Semantics}

We start with Corcoran's semantic system for D, based on families of nonempty sets (see [7], Section 3, pp. 103ff). Let $M=\left\{U_{i}\right\}_{i \in I}$ be a family of nonempty sets with non-empty index set $I$, and let $g: V \rightarrow M$. We define the interpretation function $\llbracket \cdot \rrbracket_{M, g}: \mathcal{P}_{V} \rightarrow\{0,1\}$ as follows:

\section{Definition 3}

1. $\llbracket x a y \rrbracket_{M, g}=1$ if and only if $g(x) \subseteq g(y)$,

2. $\llbracket x e y \rrbracket_{M, g}=1$ if and only if $g(x) \cap g(y)=\emptyset$,

3. $\llbracket x i y \rrbracket_{M, g}=1$ if and only if $g(x) \cap g(y) \neq \emptyset$,

4. $\llbracket x o y \rrbracket_{M, g}=1$ if and only if $g(x) \nsubseteq g(y)$,

The soundness proof is straightforward. The completeness proof can be found in $[5] \cdot{ }^{27} \mathrm{We}$ shall refer to the class of interpretation functions based on families of non-empty sets as CSet.

With respect to Corcoran's semantic system, two kinds of claims must be kept apart: (a) exegetical claims and (b) claims about the adequacy of the semantics. With respect to (a), Corcoran esteems that truth values of sentences are 'determined extensionally', ${ }^{28}$ and this gives him good reasons to maintain that the interpretation of terms should be given by non-empty sets. ${ }^{29}$

\footnotetext{
${ }^{27}$ From the previous definitions it is not hard to see that there is no $d \in \mathcal{P}_{V}$ such that $\emptyset \models d$. In other words, there is no $d$ such that $\llbracket d \rrbracket_{M, g}=1$ for every $g$ and every $M$. However, if we were to leave out the requirement that $x \neq y$ in the definition of $\mathcal{P}_{V}$, we would have $\emptyset \models x a x$ and $\emptyset \models x i x$, since $g(x) \subseteq g(x)$ and $g(x) \cap g(x) \neq \emptyset$ both hold for any $g: V \rightarrow M$ and any $M$. This is precisely why it was said in footnote 25 that the issue of reflexive sentences is important from a mathematical perspective. But in fact, it is not difficult to solve the problem: it is sufficient to introduce a new 'empty' rule with which we can produce the reflexive sentence xax from an empty set of premises - see [18].

${ }^{28}$ The full quote is:"Aristotle regarded the truth-value of the non-modal categorical sentences as determined extensionally (Prior Analytics, 24a26 ff)" [7, p. 103].

${ }^{29}$ With respect to non-empty sets: "Since Aristotle held that every secondary substance must subsume at least one primary substance (Categories, 2a34-2b7) ... [, then $g]$ is a function which assigns a non-empty set to each member of [V]" [7, p. 104]. See also his note 13 on the issue of existential import.
} 
The adequacy claim for this semantic system (issue (b)) might be based on a number of observations. First, it is standard mathematical practice to define mathematical objects and structures in terms of set theory. Second, such an extensional interpretation fits in well with the traditional semantic interpretation of syllogisms in terms of Venn diagrams. Third, for many terms, though not for all, it makes sense to conceive of their meaning as corresponding to sets of entities. So indeed, this extensional interpretation of syllogistic is in many senses quite plausible. However, in Section 5 we will argue that it is not uncontroversial.

\subsubsection{Martin's Semantics}

But of course, $C$ Set is not the only class of interpretation functions with respect to which D is sound and complete. It is equally possible to define a class of interpretation functions based on meet semi-lattices in such a way that D is sound and complete with respect to it, as proposed by John Martin [19].

In order to obtain a class of interpretation functions based on meet semilattices it is sufficient to replace Definition 3 with Definition 4:

Definition 4 Let $\langle L, \wedge, \boldsymbol{0}\rangle$ be a bound meet semilattice with $\mathbf{0}$ as its least element, $M=L-\{\mathbf{0}\}$, and $g: V \rightarrow M$. The interpretation function can now be defined as follows:

1. $\llbracket x a y \rrbracket_{M, g}=1$ if and only if $g(x) \wedge g(y)=g(x)$,

2. $\llbracket x e y \rrbracket_{M, g}=1$ if and only if $g(x) \wedge g(y)=\mathbf{0}$,

3. $\llbracket x i y \rrbracket_{M, g}=1$ if and only if $g(x) \wedge g(y) \neq \mathbf{0}$,

4. $\llbracket x o y \rrbracket_{M, g}=1$ if and only if $g(x) \wedge g(y) \neq g(x)$,

We shall refer to the class of such interpretation functions as CLat. It has been proved that $\mathrm{D}$ is sound and complete with respect to CLat [18]. The adequacy of this semantic system can be argued for on the basis of a few observations.

Martin's [19] focus is on the notion of abstraction, which he views as equally crucial for both Aritstotelian and Neoplatonic logic. ${ }^{30}$ However, there are substantial differences between how each of these traditions interprets the notion of abstraction. Martin thinks that the best way to analyze the differences is algebraically: he claims that underlying the notion of abstraction is the notion of order, which can be captured algebraically. This is why Martin is interested

\footnotetext{
30 "From its earliest days in logic, abstraction has been closely tied to concepts of order and to various unary operations interpreting negation ... This collection explores this notion of order and its related operations" $[19$, p. xi].
} 
in meet semi-lattices: his interest stems from the fact that they are the minimal algebraic structures with which an order relation can be defined. ${ }^{31}$

Indeed, the concept of abstraction seems to be at the core of syllogistic. For example, we can view the truth of xay categorical sentences as determined by the increasing order of abstraction between $x$ and $y$. (In fact, a similar idea seems to underlie Leibniz' analysis of categorical sentences-see below). Support for the view that the notion of validity of categorical syllogistic seems to rely on the properties of abstraction comes from the observation that the ordering determined by abstraction is inversely related to the sets of objects to which the terms apply. ${ }^{32}$ In fact, the entire syllogistic system can be accounted for by means of very basic principles of monotonicity, ${ }^{33}$ which again reinforces the idea that order is the crucial concept. Finally, the class of meet semi-lattices captures the most general framework that can deal with order relations; such a framework can be fruitfully exploited to account for the concept of ecthesis and for the problem of existential import of categorical sentences [19, ch. 2].

\subsubsection{Leibniz's Semantics}

One question prompted by the framework proposed in [18] is whether the class of interpretation functions appropriate for D is composed exclusively of interpretations that are instances of Definition 4. The answer to this question is negative: there are several other classes of interpretation functions with respect to which $\mathrm{D}$ is sound and complete, but which are not contained in Clat, as we will show in Section 4.3.

One such class can be built on the basis of Leibniz's 'characteristic numbers'. Leibniz made several attempts to formulate interpretations for Aristotelian syllogistic. The one presented here is the most interesting one (see, for instance, On Characteristic Numbers in [14]), for which a completeness proof has been given [4]. The interpretation function for this class, adapted from Definition 1.3 .1 of $[4$, p. 545], is the following:

Definition 5 Let $M \subseteq \mathbb{N}^{2}$ be such that if $(n, \alpha) \in M$, then $n$ and $\alpha$ are coprime. We call any such set a set of characteristic numbers. Let $g: V \rightarrow M$. Let us

\footnotetext{
31 "Though it diverges from the linear notion of the Pythagorean-Neoplatonists, abstraction in Aristotle's theory of definition is also algebraic. As developed by Porphyry in the Isagoge and by others in the Middle Ages, Aristotle's theory holds that a difference combines with a genus to define a species. The process may be viewed algebraically. There is a function, essentially a lattice meet operation $\wedge$, that is defined on pairs consisting of a difference $D$ and a genus $G$ to produce a value that is a species $S: D \wedge G=S$. As will become clear, $\wedge$ possesses the minimal properties necessary for defining an order in the usual way" [19, p. xiii].

32 "Moreover, although the definitional intension of the genus is literally part of that of the species, the species as a set of objects in the world is a subset of the genus viewed as a set" [19, p. 39].

${ }^{33}$ As also suggested by the foundational role attributed to the principles 'predicated-of-all' and 'predicated-of-none' by Aristotle himself.
} 
suppose that $g(x)=(n, \alpha)$ and $g(y)=(m, \beta)$. The interpretation function can now be defined as follows:

1. $\llbracket x a y \rrbracket_{M, g}=1$ if and only if $m \mid n$ and $\beta \mid \alpha$,

2. $\llbracket x e y \rrbracket_{M, g}=1$ if and only if either $n$ and $\beta$, or $m$ and $\alpha$ are not coprime,

3. $\llbracket x i y \rrbracket_{M, g}=1$ if and only if $n$ and $\beta$ are coprime and so are $m$ and $\alpha$,

4. $\llbracket x o y \rrbracket_{M, g}=1$ if and only if either $n \backslash m$ or $\beta \backslash \alpha$.

We shall call CLeib the class defined by Definition 5. The adequacy for this semantic system may be argued for with a few observations. To begin with, and as mentioned above, there seems to be a connection between categorical sentences of the form xay and the 'degree' of abstraction between $x$ and $y$. This is neatly captured by Leibniz in terms of characteristic numbers. That two numbers $x$ and $y$ are $a$-related means that the characteristics of $y$ are part of the characteristics of $x$-i.e. the characteristic numbers of $y$ are divisors of the characteristic numbers of $x$. This in turn neatly reflects Leibniz' own metaphysical views in terms of monads and their attributes.

Moreover, recall that Aristotle's project (as interpreted by Corcoran) is essentially epistemological, that is, to show that a given syllogism is valid. This epistemological component is Leibniz' leading motivation for developing the system of characteristic numbers. In fact, once characteristic numbers for terms are found, resolving a syllogism is no more than performing a number of arithmetical operations, a calculation. ${ }^{34}$ Thus, the certainty of valid syllogistic steps can be compared to the certainty of arithmetic. ${ }^{35}$

Another point brought up by Leibniz to motivate his system is the generality of numbers, and how they can be applied across the board to any subject matter. In a sense, this gives numbers a pride of place as an epistemological tool, thus suggesting that a semantic system for syllogistic based on (pairs of) numbers is perfectly 'adequate'. ${ }^{36}$

\footnotetext{
34 “... [A] calculus that deals with subject matter can be separated from a formal calculus. For although I discovered that one can assign a characteristic number to each term or notion (with whose help to calculate and to reason will, in the future, be the same) in fact, on account of the marvelous complexity of things, I cannot yet set forth the true characteristic numbers ... Nevertheless, I reflected, the form of inferences can be dealt with in a calculus and demonstrated with fictitious numbers." [14, p. 10-11] See also [17].

35 "For although people can be made worse off by all other gifts, correct reasoning alone can only be for the good. Moreover, who could doubt that reasoning will finally be correct, when it is everywhere as clear and certain as arithmetic has been up until now?" [14, p. 8]

36 "There is an old saying that God made everything in accordance with weight, measure, and number. But there are things which cannot be weighted, namely, those that lack force and power [vis ac potentia], and there are also things that lack parts and thus cannot be measured. But there is nothing that cannot be numbered. And so number is, as it were, metaphysical shape, and arithmetic is, in a certain sense, the Statics of the Universe, that by which the powers of things are investigated" $[14$, p. 5].
} 


\subsubsection{A Semantics Induced by $D$}

Another class of interpretation functions appropriate for $\mathrm{D}$ is what we shall call CMod. This class consists of all the interpretation functions based on first-order models of a theory called $T_{R D}$ [1], which is directly induced from D. This theory has been used to prove the minimality of RD [1] and to clarify the relations existing between D and Łukasiewicz's formalization of Aristotelian logic [2]. The theory is defined as follows: we use a similarity type $\tau=\{A, E\}$ without equality symbol, ${ }^{37}$ with only two binary relations. Let the set of variables of the language be equal to $V$.

We call $T_{R D}$ the theory defined by the following axioms, which are straightforward translations of rules (I)-(IV) of the deductive system:

$$
\begin{array}{ll}
A_{1} & \forall x, y(x E y \leftrightarrow y E x) \\
A_{2} \quad \forall x, y(x A y \rightarrow \neg(x E y)) \\
A_{3} \quad \forall x, y, z(y A z \wedge x A y \rightarrow x A z) \\
A_{4} \quad \forall x, y, z(y E z \wedge x A y \rightarrow x E z)
\end{array}
$$

Definition 6 Let $\mathcal{M}=\left\langle M, A^{\mathcal{M}}, E^{\mathcal{M}}\right\rangle$ be a model of $T_{R D}$ and $g: V \rightarrow M$. The interpretation function can now be defined as follows:

1. $\llbracket x a y \rrbracket_{M, g}=1$ if and only if $\mathcal{M} \models x A y[g]$,

2. $\llbracket x e y \rrbracket_{M, g}=1$ if and only if $\mathcal{M} \models x E y[g]$,

3. $\llbracket x i y \rrbracket_{M, g}=1$ if and only if $\mathcal{M} \forall x E y[g]$,

4. $\llbracket x o y \rrbracket_{M, g}=1$ if and only if $\mathcal{M} \not x x A y[g]$,

D's soundness with respect to CMod follows from the soundness of rules (I)-(IV), and this in turn follows from the fact that every interpretation function comes from a model of $T_{R D}$. The completeness proof can be found in [2]. In a sense, CMod is not very 'adequate', as it is essentially induced from the deductive system itself; but it has been useful to prove general properties of syllogistic and formalizations thereof. So while it may not be the most conceptually entrenched of the semantic systems discussed here, it is not obvious on what grounds it could be excluded as a legitimate semantic system for syllogistic. Indeed, CMod is based on first-order models, which are widely used to formulate semantic systems for a variety of logics. In fact, many seem to view first-order models as the most adequate class of mathematical structures for the purposes of formulating a semantics for any given (first-order) language (see Section 5).

Now that we have at hand a number of classes of interpretation functions, that is, a number of different semantic systems for $\mathrm{D}$, we can investigate

\footnotetext{
${ }^{37}$ The consequence of leaving out the equality symbol is that the atomic sentences are only of the form $x A y$ or $x E y$.
} 
whether there is some structure among these classes, i.e. whether some classes are subclasses of others (Section 4.3).

\subsection{Finding Some Structure}

So far we have four classes of interpretation functions with respect to which $\mathrm{D}$ is sound and complete. These are CSet, CLat, CLeib, and CMod. What are the relations among them? The first step to address this question is to note that if $\mathcal{C}_{1}$ and $\mathcal{C}_{2}$ are two classes of interpretation functions with respect to which $\mathrm{D}$ is sound and complete, and if $\llbracket \cdot \rrbracket_{M, g} \in \mathcal{C}_{1}$, then there exists $\llbracket \cdot \rrbracket_{M, g}^{\prime} \in \mathcal{C}_{2}$ such that $\llbracket d \rrbracket_{M, g}=\llbracket d \rrbracket_{M, g}^{\prime}$, for every $d .^{38}$ That is, they all provide the same truthpreserving patterns, as is to be expected from their respective completeness theorems (but of course, only as long as the arguments considered are exclusively formulated in the language underlying D).

These classes are all different from one another, however, in that the truth conditions they assign to categorical sentences are provided by different structural information in each case. For example, let $M=\wp(S)-\{\emptyset, S\}$ be the family of all subsets of a given set $S(|S|>1)$ that are different from $\emptyset$ and $S$, and let $g: V \rightarrow M$. Starting from these $M$ and $g$ we can define truth conditions for categorical sentences on the basis of the inclusion relation. The interpretation function thus defined, which we will call $\llbracket \cdot \rrbracket_{M, g}$, belongs to $C S e t$. On the other hand, if we consider the same interpretation function as a member of CLat, it will provide truth conditions on the basis of the meet operator, which is obtained from the operation of intersection between sets. This is not, however, the only lattice that can be accommodated to such $M$ and $g$. By taking the reciprocal of the subset relation on $M \cup\{S\}$ we obtain a different meet semi-lattice, where $S$ is its least element. Call this lattice $M \downarrow$. Hence, $\llbracket \cdot \rrbracket_{M \downarrow, g}$ belongs to CLat and it assigns truth conditions to categorical sentences on the basis of the same $M$ and $g$ as $\llbracket \cdot \rrbracket_{M, g}$, but according to different structural information. Of course, the truth conditions that $\llbracket \cdot \rrbracket_{M, g}$ and $\llbracket \cdot \rrbracket_{M \downarrow, g}$ define are quite different. For example, by Definitions 3 and 4 it follows that, for any $x$ and $y$ such that $g(x) \neq g(y), \llbracket x a y \rrbracket_{M, g}=1$ iff $\llbracket x a y \rrbracket_{M \downarrow, g}=0$.

The upshot of these considerations is, firstly, that we must always bear in mind the superindexes $\mathbf{A}$ and $\mathbf{E}$ for each interpretation function, as they bring out the structural information according to which truth conditions are defined. Secondly, we can immediately observe a difference between CSet and CLat. On the one hand, $\llbracket \cdot \rrbracket_{M \downarrow, g}$ is a member of CLat that is not a member of CSet.

\footnotetext{
${ }^{38}$ This follows straightforwardly from the fact that all these classes are such that D is sound and complete with respect to them. Therefore, given $\llbracket \cdot \rrbracket_{M, g}$ in one of them, the set $K:=\left\{d: \llbracket d \rrbracket_{M, g}=\right.$ $1\}$ is maximally consistent, and hence it has a true interpretation in all the other classes.
} 
Moreover, given that not all families of non-empty sets are such that their inclusion relation allows us to obtain a meet operator from the operation of intersection, there are members of CSet that are not members of CLat. A simple example is the following:

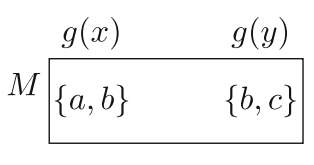

$\emptyset$

The sets $\{a, b\}$ and $\{b, c\}$ have a non-empty intersection, $\{b\}$, which does not belong to the family $M$. A fortiori, $M$ is not a meet semi-lattice.

This does not mean that we cannot find a lattice structure over such $M$ and $g$. We can, but the meet operator to be found would not coincide with the operation of intersection. This is a case parallel to $\llbracket \cdot \rrbracket_{M, g}$ and $\llbracket \cdot \rrbracket_{M \downarrow, g}$ mentioned above. As a consequence, we have shown that CSet and CLat are not included in one another, yet their intersection is non-empty $\left(\llbracket \cdot \rrbracket_{M, g}\right.$ belongs to both classes).

Similar conclusions can be drawn concerning the classes CLat and CLeib. Some interpretation functions in the latter class could be shown to have a meet semi-lattice structure, such as the following:

Take $1<N \in \mathbb{N}$ and let the set of characteristic numbers be $M:=\left\{\left(2^{n}, 3^{n}\right)\right.$ : $0<n<N\} .{ }^{39}$ Define $\langle L, \wedge, \mathbf{0}\rangle$ as follows:

- Let $\mathbf{0}$ be an arbitrary symbol.

- Define $\left(2^{n}, 3^{n}\right) \wedge\left(2^{m}, 3^{m}\right):=(\max \{n, m\}, \max \{\alpha, \beta\})$, and define $\left(2^{n}, 3^{n}\right) \wedge$ $\mathbf{0}:=\mathbf{0}$.

- $L=M \cup\{\mathbf{0}\}$.

It is not hard to see that $L$ is a meet semi-lattice inducing the right ordering relation. That is, if $\llbracket \cdot \rrbracket_{M, g}$ is the interpretation function in CLeib based on $M$ and $g$ above, then for all $x, y$ :

$$
\llbracket x a y \rrbracket_{M, g}=1 \quad \text { iff } \quad g(x) \wedge g(y)=g(x),
$$

and

$$
\llbracket x e y \rrbracket_{M, g}=1 \quad \text { iff } \quad g(x) \wedge g(y)=\mathbf{0} .
$$

\footnotetext{
${ }^{39}$ Since the completeness proof for CLeib requires $V$ finite, we cannot take $n$ to range over all $\mathbb{N}$. From this example, it can be seen that the order always has a maximum, and therefore, the construction presented in [4] cannot handle infinite cases such as $K=\left\{x_{1} a x_{2}, x_{2} a x_{3}, \ldots\right\}$.
} 
Moreover, there are also interpretation functions in CLeib that are not in $C$ Lat. Consider the following counterexample $M$, similar to the counterexample in the case of CSet:

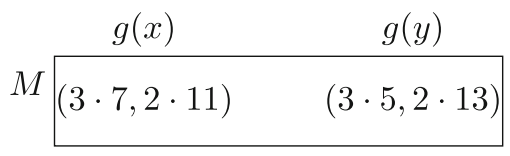

0

The pair $(3,2)$ stands in the $a$ relation to both $(3 \cdot 7,2 \cdot 11)$ and $(3 \cdot 5,2 \cdot 13)$ according to Definition 5, yet it does not belong to $M$. A similar reasoning as above can show that any meet-semilattice structure we can find in $L$ will not coincide with the one obtained from the $a$ relation. ${ }^{40}$

There is no relation of inclusion between CSet and CLeib, because the interpretation functions in each class are based on structures that are altogether different: sets, in the case of CSet, and pairs of numbers, in the case of CLeib.

Finally, we can show that the three classes CSet, CLat and CLeib are subclasses of CMod. In fact, we can show that any class of interpretation functions with respect to which $\mathrm{D}$ is sound and complete will be a subclass of $C M o d$. Let $C$ be a class of interpretation functions. If $\mathrm{D}$ is sound with respect to $C$, any interpretation $\llbracket \cdot \rrbracket_{M, g}^{\mathbf{A}, \mathbf{E}} \in C$ must make valid all the rules of D. Therefore, the model $\mathcal{M}=\langle M, \mathbf{A}, \mathbf{E}\rangle$ turns out to be a model of $T_{R D}$. Given that the interpretation function that we construct from $\mathcal{M}=\langle M, \mathbf{A}, \mathbf{E}\rangle$ and $g$ is the same as $\llbracket \cdot \rrbracket_{M, g}^{\mathbf{A}, \mathbf{E}}$, it follows that $C$ is a subclass of $C M o d$. This means that $C M o d$ is the largest class that $\mathrm{D}$ is sound with respect to. Since $\mathrm{D}$ is complete with respect to CMod, we obtain that CMod is the largest class of interpretations with respect to which D is sound and complete-which is not surprising, as CMod was induced from D.

\footnotetext{
${ }^{40}$ Since this fact is less trivial that in the case of $C S e t$, we give here a full proof: Suppose towards contradiction that there is a meet semilattice $\langle L, \wedge, \mathbf{0}\rangle$ such that $M=L-\{\mathbf{0}\}$. Also, suppose that for all $x, y$ :

$$
\llbracket x a y \rrbracket_{M, g}=1 \quad \text { iff } \quad g(x) \wedge g(y)=g(x),
$$

and

$$
\llbracket x e y \rrbracket_{M, g}=1 \quad \text { iff } \quad g(x) \wedge g(y)=\mathbf{0} .
$$

Now, following the diagram above with the obvious assignment $g$, we have that $\llbracket x a y \rrbracket M, g=0$ and $\llbracket y a x \rrbracket_{M, g}=0$. Therefore, $g(x) \wedge g(y) \neq g(x)$ and $g(x) \wedge g(y) \neq g(y)$ [recall that $\wedge$ is commutative]. It follows from here, and from the requirement that $\wedge$ be the meet of a semilattice, that $g(x) \wedge$ $g(y)$ should be an element of the lattice. But since it cannot be either $g(x)$ or $g(y)$, it must be 0. Therefore, $\llbracket x e y \rrbracket_{M, g}=1$. On the other hand, by definition of $e$ in Definition 5, $\llbracket x e y \rrbracket_{M, g}=0$ [because the opposite coordinates in both characteristic numbers are coprime]. We have reached a contradiction. Therefore, there is no meet-semilattice over $M$ satisfying the equations above.
} 
That CMod is strictly larger than CLat follows from the existence of an interpretation $\llbracket \cdot \rrbracket_{M, g}$ such that $\llbracket \cdot \rrbracket_{M, g} \in C M o d$, but $\llbracket \cdot \rrbracket_{M, g} \notin C$ Lat. We can use here the following model $\mathcal{B}=\left\langle M, A^{\mathcal{B}}, E^{\mathcal{B}}\right\rangle$ :

$$
\begin{aligned}
M= & \left\{b_{1}, b_{2}, b_{3}, b_{4}\right\} \\
A^{\mathcal{B}}= & \left\{\left(b_{1}, b_{2}\right),\left(b_{2}, b_{1}\right),\left(b_{1}, b_{3}\right),\left(b_{3}, b_{1}\right),\left(b_{2}, b_{3}\right),\left(b_{3}, b_{2}\right),\right. \\
& \left.\left(b_{1}, b_{1}\right),\left(b_{2}, b_{2}\right),\left(b_{3}, b_{3}\right),\left(b_{4}, b_{4}\right)\right\} \\
x E^{\mathcal{B}} y \Longleftrightarrow & x A^{\mathcal{B}} y \text { does not hold }
\end{aligned}
$$

This model can be represented in the following diagram:

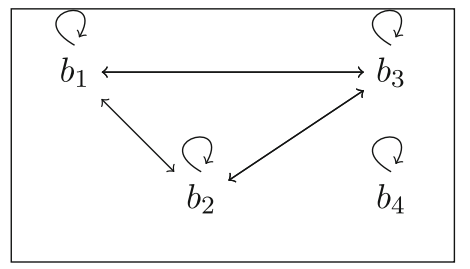

$\mathcal{B}$

We also need an assignment function $g: V \rightarrow M$ in order to construct an interpretation function $\llbracket \cdot \rrbracket_{M, g}$. We assume that $g(x)=b_{2}$ and $g(y)=b_{3}$. Clearly $\llbracket \cdot \rrbracket_{M, g} \in C M o d$. Now, assume towards contradiction that $\llbracket \cdot \rrbracket_{M, g} \in C$ Lat. This implies that there is a bound meet-semilattice $\langle L, \wedge\rangle$ with least element 0 such that $M=L-\{0\}$ and such that $\llbracket x a y \rrbracket_{M, g}=1$ iff $g(x) \wedge g(y)=g(x)$. On the one hand we have:

$\mathcal{B} \models x A y[g] \quad$ iff $\quad \llbracket x a y \rrbracket_{M, g}=1 \quad$ iff $\quad g(x) \wedge g(y)=g(x) \quad$ iff $\quad b_{2} \wedge b_{3}=b_{2}$

But on the other hand we have:

$$
\mathcal{B} \models y A x[g] \quad \text { iff } \quad \llbracket y a x \rrbracket_{M, g}=1 \quad \text { iff } \quad g(y) \wedge g(x)=g(y) \quad \text { iff } \quad b_{3} \wedge b_{2}=b_{3}
$$

Since the left-hand sides of Eqs. 1-2 are true, we obtain $b_{2} \wedge b_{3}=b_{2}$ and $b_{3} \wedge b_{2}=b_{3}$. Because the $\wedge$ of any meet-semilattice is commutative, we can conclude that $b_{2}=b_{3}$, which is a contradiction.

Thus, we obtain the following structure among the classes CSet, CLeib, CLat, and CMod:

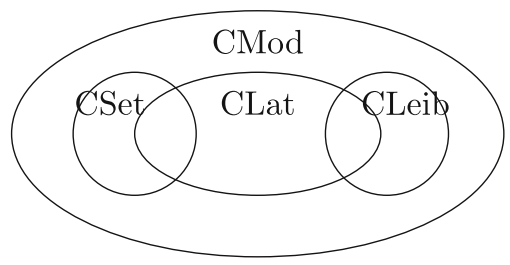




\section{Looking for an Adequate Semantics ${ }^{41}$}

\subsection{A Merely Apparent Harmony}

Thus, the four semantic systems for syllogistic presented here are not extensionally equivalent, in the sense that the structural information that the interpretation functions carry in each semantic system differs. And yet, D is sound and complete with respect to each system, which means that they all agree on what can be expressed and proved in D. But we cannot assume that D successfully captures (in the sense of being extensionally equivalent) Aristotle's general definition of deduction (even for the restricted fragment of the language in question), as this is precisely what must be established. (This was Corcoran's very goal in his formalization.) So it might well be that the disagreements between the different semantics are in fact substantive for the informal notion of (in)validity after all, but that such facts cannot be expressed in the very simple language underlying $\mathrm{D}$. In other words, the apparent agreement between the four semantic systems which follows from the fact that $\mathrm{D}$ is sound and complete with respect to each of them is very likely just a consequence of the limited expressive power of the underlying language.

Given that D is 'blind' to the differences between the different semantic systems, these differences may become apparent only with a richer, more expressive language. But should we be concerned with such differences, given that they do not appear to be relevant from the point of view of the fragment of the language we are interested in? Well, we should, precisely because the informal notion of validity (deduction) that is the starting point of the whole enterprise-its target phenomenon-is not restricted to a given language; it is meant to be perfectly general.

Moreover, the language underlying $\mathrm{D}$ is very limited indeed. In the history of logic, several proposals to extend the basic categorical language have been put forward; in particular, logicians have investigated the logical properties of extended categorical languages including negative terms (e.g. 'non-man'), singular terms and modalities. (Such developments are particularly conspicuous in the later Latin medieval tradition.) Thus, extending the categorical language is in no way a 'historical abnormality'. And yet, from the point of view of such extended languages, the four semantic systems presented here no longer agree, even for very simple extensions. It is known for instance [12] that Leibniz's semantic system in terms of pairs of numbers no longer functions once negative terms are introduced. Hence, the extended deductive system with negative terms is no longer sound and complete with respect to semantic system CLeib. But the deductive system formulated with this extended language is sound and

\footnotetext{
${ }^{41}$ Notice the use of an indefinite article: we do not contend that there is necessarily one single adequate semantics in each case.
} 
complete with respect to $\mathrm{CSet} .{ }^{42}$ Similarly, if the language is extended so as to allow for reflexive sentences, then the extended deductive system will not be sound and complete with respect to $C S e t$, as already mentioned, but it will be sound and complete with respect to CMod. ${ }^{43}$

In other words, by enriching the language, the apparent harmony between the four semantic systems in question is disrupted. They no longer agree on the class of arguments deemed valid (for the extended language), and thus can no longer all be sound and complete with respect to the new deductive system. Therefore, clearly they cannot all be adequate semantic systems for extended syllogistic systems, which also means (if the extension of the language is conservative) that they were not all adequate in the first place; but the limited language of categorical arguments is not sufficiently fine-grained to 'see' this. Recall that we are here interested in the cogency of premise (2) of the squeezing argument, i.e. in the connections between the target phenomenonan informal notion of validity-and semantic counterparts in terms of mathematical structures. This connection should not depend on how expressive the language underlying a given deductive system is, as a specific language is not a relevant element for this relation. But by increasing the expressive power of the language underlying the deductive system in question, we realize that the four semantic systems in fact disagree substantially with each other on how best to characterize the target phenomenon.

\subsection{Etchemendy on Choosing a Semantics}

At this point, we must ask ourselves what it takes for a semantics to be an adequate semantics, or in other words, what it is that a semantics is trying to capture or to be a model of. J. Etchemendy and S. Shapiro are among those who have discussed this issue. Etchemendy has famously criticized what he views as a 'Tarskian' notion of logical consequence, which he characterizes as an 'interpretational' approach ${ }^{44}$ in contrast with his preferred 'representational' approach. ${ }^{45}$ One of the features of the interpretational approach as described by Etchemendy is its language dependence: the meaning of a compound expression is given by the semantic values of its parts, and the interpretational procedure is entirely dependent on the logical vs. non-logical partition, which typically varies from language to language. Now, insofar as we here endorse the idea that the connection between a semantics and a target phenomenon should not be language-dependent, we are also interested in a 'representational' approach to model-theory and formal semantics

\footnotetext{
${ }^{42}$ The negation operator on terms can be easily interpreted by means of the complement operation on sets with respect to the union of the family of non-empty sets.

${ }^{43}$ For details, see [2].

${ }^{44} \mathrm{On}$ this approach, the focus is on the language in which the relation of logical consequence is expressed: a sentence is a logical consequence of a set of sentences iff on every interpretation of their non-logical vocabulary, whenever the premises are true, so is the conclusion.

${ }^{45}$ On how Etchemendy understands this notion, see quote just below.
} 
more generally. But what is the rationale behind designing a semantics for a given language according to the representational approach? Here is what Etchemendy says on the matter:

I called this view of model theory 'representational' because the settheoretic structures are seen as full-fledged representations: models of the world. [...] According to the representational view, our goal in constructing a semantics is to devise a class of models that represents all logically possible ways the world might be that are relevant to the truth or falsity of sentences in the language, and to define a relation of truth in a model that satisfies the following constraint: a sentence $\phi$ should be true in model $\mathcal{M}$ if and only if $\phi$ would be true if the world were as depicted by $\mathcal{M}$, that is, if $\mathcal{M}$ were an accurate model. The models are designed to represent the world in a particularly straightforward way, and this is important. Any individual model represents a logically possible configuration of the world and any two (non-isomorphic) models are logically incompatible: at most one can be accurate. But jointly, they are meant to represent all of the possibilities relevant to the truth values of sentences in the language. In other words, if we've designed our semantics right, the models impose an exhaustive partition on the possible circumstances that could influence the truth of our sentences. $[10,287]$ (emphasis added)

Of course, Etchemendy does not claim that model-theory offers a perfectly faithful picture of the world and of ways the world might be; model-theoretic structures are simplified representations, picking out only the elements that are relevant for the truth-value of sentences. Still, that model-theory can offer representations of ways the world might be, and what is more, that it can exhaust the class of these possibilities, is a very strong claim indeed. Why is it that the particular structure of model-theoretic constructions is an adequate tool to represent the world? This is a substantive quasi-metaphysical claim, and while Etchemendy is well aware of its substantiveness (see footnote 19, $[10,288])$, in his essay he "set[s] aside [this] important question". What if the actual deep structure of the world is not as model-theory assumes it to be? This could well be the case.

Etchemendy does not mention alternative semantic systems for first-order logic, e.g. the one provided by category theory [16]. But one may wonder why he (presumably) considers model-theory to be more adequate as a semantics for first-order logic than these alternatives, i.e. as a tool to represent logically possible configurations of the world. ${ }^{46}$ In effect, against the background of a situation where there is more than one viable alternative as a semantics for

\footnotetext{
${ }^{46}$ As pointed out by an anonymous referee, the problem is not just Etchemendy's-it is (almost) everybody's problem. The adequacy of model-theory as a representation of logically possible configurations of the world is indeed a crucial and complex matter, but for reasons of space a detailed discussion of it cannot be offered here.
} 
a logic (in view of a given target phenomenon), it becomes patent that they cannot all be equally good representations of the class of logically possible configurations of the world. Just as there are different, competing theories of the physical world in physics which cannot all be 'right', if what a semantics does is to represent and depict possible configurations of the world, then different semantics will offer different and possibly incompatible accounts of the ways the world can be.

Indeed, the four semantic systems for syllogistic presented here presuppose, or at least suggest, rather different metaphysical positions: CSet could be viewed as relying on an 'Aristotelian' metaphysical conception of the world (in the sense of the ontology presented in the Categories); CLat suggests a (neo-)Platonic view of reality; CLeib is particularly compatible with Leibniz' own conception of a world made of monads containing infinite attributes; and CMod, insofar as it is based on first-order models, relies on the assumption that model-theory is a privileged way of representing the world. Now, they cannot possibly all be equally adequate; on this view of what it means to formulate a semantics for a logic, they are ultimately competing views on the nature of metaphysical reality. And even if what is at stake is only how the truth of propositions is affected, they clearly do not agree with each other either. They agree with each other with respect to sentences in the original categorical language, but they begin to disagree as soon as the language is extended.

If we follow Etchemendy's account, when we do formal semantics, i.e. when we design a semantics for a given logic, we are (or should be) in fact engaging in substantive metaphysical investigations. Now, these investigations should of course satisfy the standards of philosophical rigor to be recommended for this kind of inquiry. As it stands, however, Etchemendy's analysis simply states that model-theory aptly represents different possible configurations of reality without getting into the details of why the fitting is (presumably) so successful; further argumentation would be required so as to establish this thesis (as $\left[10,288\right.$, fn. 19] realizes himself) ${ }^{47}$ Clearly, the choice of a given class of mathematical structures as the semantics for a given logic entails a form of ontological commitment.

\subsection{Shapiro: What is a Semantics a Model of?}

Shapiro makes similar suggestions in [20] and [21]. He introduces the concepts of a semantics being 'correct' and 'adequate', taking the class of valid arguments in natural language as the yardstick against which these properties

\footnotetext{
${ }^{47}$ For example, if borders in the world happen to be fuzzy and vague rather than sharp and clear-cut (i.e. the thesis of metaphysical vagueness), then model-theory is clearly a bad model of metaphysical reality. It presupposes that an object either belongs or does not belong to a given class, i.e. it presupposes sharp boundaries. But again, for reasons of space, we are unable to offer a detailed discussion of reasons why model-theory may be a bad model of metaphysical reality.
} 
are established for a given semantics. ${ }^{48}$ Premise (2) of the squeezing argument corresponds to Shapiro's notion of a semantics being 'adequate'. Shapiro raises a version of the very question we are concerned with here: "What is modeltheoretic semantics a model of?" (But notice that we here do not restrict ourselves to model-theory; we also consider other kinds of mathematical structures that can be used to formulate a semantics for a logic.) The gist of his reply to this question is essentially in the spirit of Etchemendy's views:

From this perspective, an interpretation in the model theory represents a way the world might be-a possible world - and the relation of satisfaction tells us which formulas would be true, had the world been that way. $[21,663]$

But what does he mean by 'a way the world might be'? Here, Shapiro makes an interesting but controversial claim:

What does matter for model-theoretic consequence is the size of each interpretation. The class of interpretations represents the range (or a range) of sizes the universe might be. I submit that this is the only modality that is registered in model-theoretic consequence. [21, 663]

$\mathrm{He}$ argues for this claim on the basis of the isomorphism property common to "any model-theoretic semantics worthy of the name". But if this is true, then model-theoretic semantics is in fact a rather limited tool for representing possibilities, i.e. ways the world might be. Arguably, it severely undergenerates with respect to possibilities, as it conflates different possibilities which may be genuinely different but which happen to have the same 'size'. It may also overgenerate, at least from the point of view of a strict finitist for example, if it allows for possibilities of infinite sizes (see comments on the consequences of a semantics that undergenerates or overgenerates below). Shapiro, however, takes model-theoretic semantics to be 'adequate', i.e. to validate premise (2) of the squeezing argument:

In other words, the model-theoretic semantics is adequate if each interpretation in the semantics corresponds to a genuinely possible world. In light of the isomorphism property, all that adequacy requires is that each interpretation represents a possible size of the universe. [...] I take it for granted that the interpretations of standard model theory are indeed legitimate possibilities for first- and higher-order languages, and so for these languages at least, model-theoretic consequence is adequate. [21, 664] (emphasis added)

Now, as already suggested, we believe that this kind of optimism is directly related to the fact that alternatives to model-theoretic semantics for first- and

\footnotetext{
${ }^{48}$ Of course, the issue is that 'valid arguments in natural language' is an extremely elusive and unstable class; this is why, among other reasons, we do not refer to such a class when speaking of our target phenomenon, and instead take Aristotle's 'informal' but thoroughly 'theoretical' definition of a valid deduction as our starting point.
} 
higher-order logics are not sufficiently taken into account. This is at least to some extent a historical-sociological fact; it is quite plausible that other classes of mathematical structures would be equally 'adequate' following Shapiro's own criteria, and yet would be significantly different from the standard modeltheoretic semantics in their representations of "ways the world might be".

\subsection{Different Ways Things Can Go Wrong for a Semantics}

Here, in contrast, we have presented four different semantic systems for syllogistic; they all agree on the extension of the class of arguments deemed valid for the restricted categorical language, but they disagree as soon as rather simple extensions of the language are undertaken (e.g. the inclusion of negative terms or of reflexive sentences). Now, these extensions of the language should in principle not affect the connection between target phenomenon and semantics, which is presumably what grounds premise (2) of the squeezing argument. So if the role of premise (2) is to supply the necessary condition(s) for something to fall under the informal concept in question, these four different semantic systems cannot be equally well-positioned for the job. And given that we do not seem to have the means to adjudicate between them, this at least suggests that, even when there is only one 'standard' semantic system for a given language (e.g. model-theoretic semantics for first-order logic), we cannot really be sure that it is adequate, i.e. that it really exhausts the class of 'ways the world might be'.

It might be argued that the 'problem' originates with the informal concept itself, which, in virtue of its fuzzy borders, does not allow for a unique set of necessary conditions to be formulated. This may well be, but in this case, the choice of a technical formulation of such necessary conditions is a theoretical, substantive choice, going beyond what is afforded by the informal concept itself. In this case, there is room for discussion on which aspects of the informal notion should prevail over others. Alternatively, one may contend that the role of a semantics when formulating a logic is not that of being a representation of different possible configurations inasmuch as relevant for the target phenomenon. But what is its role then, if it is not meant to be a model, in some sense or another, of the target phenomenon ${ }^{49}$

Going back to Aristotle's definition of a valid deduction, there is no doubt that a framework which is able to represent all possible configurations of the world would be a very convenient device. The 'follows of necessity' clause in the definition is very naturally interpreted as metaphysical necessity: in a valid argument, if things are as the premises say they are, they cannot but be as the conclusion says they are. But obviously, for a given semantic system to be

\footnotetext{
${ }^{49}$ The problem of the adequacy of a given semantics with respect to its target phenomenon is very general indeed; it does not concern exclusively semantics for first-order logic or syllogistic. To mention just one further example, the semantics of dynamic epistemic logic reflects a highly idealized and somewhat contrived conception of how human agents actually reason and cognize. Its adequacy as a model of its target phenomenon is far from uncontroversial.
} 
able to perform this function, it must represent all and only the situations that could obtain (in the sense relevant for the target phenomenon in question), and extensional inadequacy may come about in different ways:

- The semantic system may undergenerate: it may fail to represent all the possible situations, and thus may rule-in as valid arguments that are not actually valid. This situation would not affect (2), as it would not have any effect on the matter of invalidity; it would though affect purely semantic accounts of validity.

- The semantic system may overgenerate: it may represent 'situations' that are not real situations from the point of view of the target phenomenon, thus possibly ruling out as invalid (on the basis of such imaginary 'situations') arguments that are in fact valid (i.e. according to the target phenomenon). Circumstances of over-generation would immediately affect the intuitive plausibility of (2): if there are too many items in a given semantic system, we simply cannot be sure that a purported counterexample is a 'real' counterexample, and thus that S-invalidity implies I-invalidity.

- The semantic system may misrepresent the range of possibilities altogether, being a construction where nothing or hardly anything that it was meant to be a model of is still to be found. In this case it is even clearer that the plausibility of (2) is threatened; if the semantics is an aberration in some sense or another, in which sense is it really entitled to provide counterexamples to validity?

In summary, the difficulties encountered so far suggest that the semantic approach based on interpretations onto mathematical structures may simply not be suitable to account for the informal notion of validity at least in the particular case of syllogistic. In fact, this seems to hold in all cases where there is no obvious, uncontroversial interpretation of the target phenomenon onto mathematical structures from the start, in the sense that e.g. the structure of the natural numbers is the intended interpretation for Peano arithmetic. In such cases, the plausibility of premise (2) is severely weakened, and any form of squeezing argument cannot straightforwardly go through.

\section{Conclusion}

The results we present here are essentially negative: there is as of yet no uncontroversial candidate for the semantic side of a technical analysis of the notion of syllogistic validity, precisely because there are no clear guidelines or criteria of what it means for a semantics to be adequate. A positive conclusion that could be drawn is that it is perhaps best to approach syllogistic purely from a proof-theoretical point of view. Indeed, some interesting work has already been done in this direction, such as [19]. But we leave this as a suggestion rather than as a conclusion strictly following from our results.

As we have argued, the choice of a particular class of mathematical structures as the appropriate semantic rendering of a given target-phenomenon is 
as much in need of justification as the formulation of a deductive system. And if there is no such justification available, what is the significance of a proof of completeness with respect to a particular semantics? In such cases, there is no guarantee that premise (2) of the squeezing argument will hold, as there is no guarantee that the relevant target-phenomenon has been adequately captured by the technical apparatus. Now, if the squeezing argument does not go through in virtue of the absence of adequate justification for premise (2), a proof of completeness will not have the philosophical significance that it might otherwise have had.

More generally, perhaps the main conclusion to be drawn from these considerations is that we simply cannot take for granted the adequacy of any semantic system based on mathematical structures as the 'correct' semantics underlying a given informal notion (of validity or otherwise)-that is, if the mathematical structure in question was not the very starting point for the analysis. What is to ensure the adequacy of the chosen semantic system? We do not seem to have sufficiently clear criteria here. To our knowledge, only Etchemendy and Shapiro have offered sustained analyses of these issues, but clearly they still require further scrutiny.

Acknowledgements The authors would like to thank John Corcoran and two anonymous referees for helpful comments on earlier drafts.

Open Access This article is distributed under the terms of the Creative Commons Attribution Noncommercial License which permits any noncommercial use, distribution, and reproduction in any medium, provided the original author(s) and source are credited.

\section{References}

1. Andrade, E. J., \& Becerra, E. (2007). Corcoran's Aristotelian syllogistic as a subsystem of first-order logic. Revista Colombiana de Matemáticas, 41(1), 67-80.

2. Andrade, E. J., \& Becerra, E. (2008). Establishing connections between Aristotle's natural deduction and first-order logic. History and Philosophy of Logic, 29(4), 309-325.

3. Boger, G. (1998). Completion, reduction and analysis: Three proof-theoretic processes in Aristotle's prior analytics. History and Philosophy of Logic, 19, 187-226.

4. Caicedo, X., \& Martin-Maldonado, A. (2001). Completitud de dos Cálculos Lógicos de Leibniz. Theoria - Segunda Época, 16(3), 539-558.

5. Corcoran, J. (1972). Completeness of an ancient logic. The Journal of Symbolic Logic, 37(4), 696-702.

6. Corcoran, J. (1974a). Aristotelian syllogisms: Valid arguments or true universalized conditionals. Mind, 83(330), 278-281.

7. Corcoran, J. (1974b). Aristotle's natural deduction system. In J. Corcoran (Ed.), Ancient logic and its modern interpretations (pp. 85-131). Dordrecht-Holland: D. Reidel Publishing Company.

8. Corcoran, J. (2003). Aristotle's Prior Analytics and Boole's Laws of Thought. History and Philosophy of Logic, 24, 261-288.

9. Dummett, M. (2003). The justification of deduction. In Truth and other enigmas (pp. 290-318). Duckworth.

10. Etchemendy, J. (2008). Reflections on consequence. In D. Patterson (Ed.), New essays on Tarski and philosophy (pp. 263-299). Oxford: Oxford University Press.

11. Field, H. (2008). Saving truth from paradox. Oxford: Oxford University Press. 
12. Glashoff, K. (2007). On negation in Leibniz' system of characteristic numbers. Unpublished manuscript, http://www.logic.glashoff.net/Texte/negation1.pdf.

13. Kreisel, G. (1967). Informal rigour and completeness proofs. In I. Lakatos (Ed.), Problems in the philosophy of mathematics (pp. 138-171). North-Holland, Amsterdam.

14. Leibniz, G. W. (1989). Philosophical essays. Edited and translated by Roger Ariew and Daniel Garber. Hackett Publishing Company, Inc.

15. Łukasiewicz, J. (1957). Aristotle's syllogistic from the standpoint of modern formal logic. Oxford University Press.

16. Makkai, M., \& Reyes, G. (1977). First-order categorical logic. Springer lecture notes in mathematics 611. New York: Springer.

17. Marshall, D. (1977). Łukasiewicz, Leibniz and the arithmetization of the syllogism. Notre Dame Journal of Formal Logic, 18 235-242.

18. Martin, J. N. (1997). Aristotle's natural deduction reconsidered. History and Philosophy of Logic, 18, 1-15.

19. Martin, J. N. (2004). Themes in neoplatonic and Aristotelian logic: Order, negation and abstraction. Ashgate.

20. Shapiro, S. (1998). Logical consequence: Models and modality. In M. Schirn (Ed.), Philosophy of mathematics today (pp. 131-156). Oxford: Oxford University Press.

21. Shapiro, S. (2005). Logical consequence, proof-theory, and model-theory. In S. Shapiro (Ed.), The Oxford handbook of philosophy of mathematics and logic (pp. 651-670). Oxford: Oxford University Press.

22. Smiley, T. (1973). What is a syllogism? Journal of Philosophical Logic, 2(1), 136-154.

23. Smith, P. (forthcoming). Squeezing arguments. Analysis.

24. Smith, P. (2007). An introduction to Gödel's theorems. Cambridge University Press.

25. Smith, R. (Ed.) (1989). Aristotle: Prior Analytics. Hackett, Indianapolis.

26. Tarski, A. (1936/2002). On the concept of following logically. History and Philosophy of Logic, 23, 155-196. 